\title{
Familial aggregation of metabolic syndrome among the Chinese: Report from the Chin-Shan community family study
}

\author{
K.L. Chien ${ }^{\mathrm{a}, \mathrm{b}}$, H.C. Hsu ${ }^{\mathrm{b}}$, W.J. Chen ${ }^{\mathrm{c}}$, M.F. Chen ${ }^{\mathrm{b}}$, T.C. Su ${ }^{\mathrm{b}}$, Y.T. Lee ${ }^{\mathrm{b}, \mathrm{d}, *}$ \\ ${ }^{a}$ Institute of Preventive Medicine, School of Public Health, National Taiwan University, Taipei, Taiwan \\ ${ }^{\mathrm{b}}$ Department of Internal Medicine, National Taiwan University Hospital, Taipei, Taiwan \\ ${ }^{\mathrm{c}}$ Institute of Epidemiology, College of Public Health, National Taiwan University, Taipei, Taiwan \\ ${ }^{\mathrm{d}}$ Ming-Shen Healthcare, Taoyuang, Taiwan
}

Received 30 April 2006; accepted 20 September 2006

Available online 9 November 2006

\begin{abstract}
Genetic study on metabolic syndrome is a great challenge, due to its complex traits and the pleiotropic manifestation of atherosclerosis. Familial aggregation and recurrence risk ratio can provide the insight of possible genetic mechanism. The ChinShan community family study was based on adolescent probands and their relatives (1356 subjects) who were recruited from one junior high school in the community. Structured questionnaires and biochemical measures were obtained in standard procedures. Definition of metabolic syndrome was followed using the criteria defined by the third adult treatment panel, with a modification of the criteria for adolescent and Asian population. Grandmothers had the highest frequencies (70\%) in metabolic syndrome and various atherosclerotic risks. Three factors were found and thus explained $68 \%$ of the overall variance. Estimated heritability was the highest in LDL and cholesterol factor (0.36 and 0.40$)$, then blood pressure/obesity factor (0.27), and insulin resistance/ dyslipidemia (0.27). Recurrence risk ratio among siblings was 2.95 (95\% confidence interval [CI]: 1.39-6.26). The adjusted odds ratio (OR) of proband's metabolic syndrome status was 1.99 (95\% CI: 1.08-3.66). The adjusted odds ratios for the three factors for predicting metabolic syndrome were all significant, with highest risk in blood pressure/obesity factor (OR: 1.27, CI: 1.22-1.33), then insulin resistance/dyslipidemia (OR: 1.29, CI: 1.16-1.23). This study demonstrated clearly familial aggregation and recurrence risk ratio of metabolic syndrome and components among the general ethnic Chinese population in Taiwan.
\end{abstract}

(C) 2006 Elsevier Ireland Ltd. All rights reserved.

Keywords: Metabolic syndrome; Familial correlation; Sibling recurrence risk ratio

\section{Introduction}

Mechanisms of metabolic syndrome (MetS) causation are complex, and environmental factors such as lifestyle change, dietary and physical inactivity are important factors for the pathogenesis of MetS [1].

\footnotetext{
* Corresponding author. Tel.: +88622356 2038; fax: +886223920456 .

E-mail address: ytlee@ha.mc.ntu.edu.tw (Y.T. Lee).
}

Genetic studies on MetS and insulin resistance were a great challenge, due to its complex traits and the possible pleiotrophy manifestation of atherosclerosis traits. There were quantitative genetic studies on MetS and insulin resistance traits, and the Framingham family data supported the sibling recurrence risk ratios were two folds [2].

Racial difference of prevalence rates implies a genetic background for MetS. Mexican-Americans had a much higher prevalence than Caucasians in American [3], and very high prevalence was found in the South-Asian 
population such as Omen and India, in which the rates were up to $41 \%$ [4,5]. There were significant familial correlation between quantitative traits of MetS, and the correlation coefficients between siblings can reach up to 0.4 [6]. The heritability estimates of MetS components were very high, up to $70 \%$ in the data regarding twins [7], indicating strong genetic factors. Austin et al. also demonstrated clearly high heritability in MetS and insulin resistance components among Japanese-Americans [8].

Previous genetic studies on MetS have several limitations. First, neither sibling nor relative recurrence risk ratio was estimated. Sibling recurrence risk ratio is an index for genetic components of binary outcome [9]. Second, previous studies were ascertained from a diseased population, and community-based general population data was not available. Finally, difficult replication of results on genome-wide linkage analyses and candidate gene association studies implied that population heterogeneity is a difficult issue to handle, and that a homogeneous population might be a useful tool to test the genetic components of MetS. Therefore, this study was designed to explore the possible genetic components on MetS and lipid profiles.

\section{Subjects, materials and methods}

\subsection{Study design and study population}

The family study started in 1997 and was designed to recruit adolescent probands from students from the only junior high school in a local community $[10,11]$. In brief, the ChinShan community family study, based on adolescent probands and their relatives recruited from a systematic sampling of one junior high school in the local community, was designed as a family-based genetic study on atherosclerotic traits. In brief, a total of 1063 students (aged 12 years, response rate 94.6\%) agreed to participate in a general health check-up after informed consent was obtained. The study was approved by IRB in National Taiwan University Hospital. They underwent examinations for anthropometric measures, blood pressure, and lipid profiles. Probands were then selected through stratified random sampling $(n=368)$, which included the atherosclerotic risk profile measures, including total cholesterol, triglyceride, LDL-cholesterol, body mass index, systolic pressure, diastolic pressure, and HDL-cholesterol. After obtaining informed consent from the probands' family members, the same measures were performed for each family member. Family relatives, including proband's grandparents, were invited and a total of 1356 subjects were included in this family study.

Structured questionnaires on family history, lifestyle habits, and medical history were collected. Anthropometric measures such as body weight, body height and waist circumference were measured following standardized procedures. Physical examination and 12-lead electrocardiogram were performed concurrently. Blood pressure was measured in a resting position by trained medical assistants. Body mass index (BMI) was calculated as weight (in $\mathrm{kg}$ )/height (in $\mathrm{m}^{2}$ ). Waist circumference was measured by a trained examiner and determined using a measuring tape positioned at the midway between the lowest rib and the iliac crest. The measurement was made at minimal respiration status, with the tape snug but without compressing the skin.

\subsection{Blood sampling and analytic methods}

The procedures for blood sampling and analytic methods have been described previously [12]. Serum samples were stored at $-70{ }^{\circ} \mathrm{C}$ prior to the batch assay using standard enzymatic tests for cholesterol and triglycerides (Merck 14354 and 14366, Germany) and precipitation methods for HDL- and LDL-cholesterol (Merck 14993 and Merck 14992). Glucose levels were measured by enzymatic assay (Merck 3389) using an Eppendorf 5060 autoanalyzer. Insulin levels were measured by enzyme immunoassay [13]. The coefficients of variation for above measurements were $5 \%$.

\subsection{Definition of metabolic syndrome and lipid profiles}

We determined the MetS status in the adult population using criteria defined by the third adult treatment panel [14], modification of criteria for waist circumference for Asian population [15]. A participant with three of following five criteria was defined as having the MetS: (1) blood pressure of at least $130 / 85 \mathrm{mmHg}$ or treated hypertension; (2) serum triglyceride of at least $150 \mathrm{mg} / \mathrm{dL}$; (3) HDL-cholesterol of $<40 \mathrm{mg} / \mathrm{dL}$ in men and $<50 \mathrm{mg} / \mathrm{dL}$ in women; (4) fasting glucose of $110 \mathrm{mg} / \mathrm{dL}$ or more; (5) waist circumference greater than $90 \mathrm{~cm}$ in men and $80 \mathrm{~cm}$ in women. For the adolescent population (12-19 years old), the following criteria was defined: (1) blood pressure above 90th percentile value of the population; (2) serum triglyceride of at least $110 \mathrm{mg} / \mathrm{dL}$; (3) HDL-cholesterol of $<40 \mathrm{mg} / \mathrm{dL}$ in men and women; (4) fasting glucose of $110 \mathrm{mg} / \mathrm{dL}$ or more; (5) waist circumference greater than 90th percentile in the population [16]. Homeostasis model assessment (HOMA) was used to identify insulin resistance syndrome, using the formula: [fasting insulin $(\mathrm{mIU} / \mathrm{L}) \times$ fasting glucose $(\mathrm{mg} / \mathrm{dL}) \times 0.05551] / 22.5$.

\subsection{Statistical analyses}

All MetS and quantitative lipid components were described by their mean, standard deviation, specified by gender and generation. Due to the skewed distribution of triglyceride, insulin and glucose values, natural logarithm transformation was performed to get normalized distribution. Age and gender adjusted Pearson correlation coefficients were calculated among MetS, insulin resistance and quantitative lipid components. We used exploratory factor 
analysis to reduce the dimension of MetS and quantitative lipid traits. The principal component method with a varimax rotation was used and the final factors were determined by eigenvalues greater than 1.0. Adjusted factor loading and explained variance of various quantitative traits were obtained. Also, we standardized the factors to mean 100, standard deviation 10 , to make the estimated odds ratios for MetS comparable.

Familial intra-trait correlation coefficients of MetS and quantitative lipid traits between spouse, parent-offspring and sibling were estimated by using the FCOR program in SAGE [17]. We used the variance component models to estimate heritability, implemented in SOLAR software [18]. We tabulated the affected sibling members by family size, stratifying by proband MetS status, and estimated the sibling recurrence risks by the odds ratio of proband status for metabolic syndrome. Finally, we used multiple logistic regression to estimate the adjusted odds ratio and $95 \%$ confidence intervals of proband MetS status and three factors by the generalized estimating equation (GEE) model to adjust the intra-familial dependence [19].

\section{Results}

The characteristics of various MetS, insulin resistance and lipid traits among family members are described in Table 1. Grandmothers had the highest cholesterol, triglyceride, LDL-cholesterol, fasting glucose, waist circumference, body mass index and blood pressure, and the average MetS scores were the highest among grandmothers $(3.25 \pm 1.37)$, then fathers and grandfathers $(2.32,2.14$, respectively). Grandmothers had the highest frequency of MetS and various atherosclerotic risks, and nearly $70 \%$ of grandmothers studied had MetS status. Persistent significant positive correlation coefficients among cholesterol, triglyceride, fasting glucose, HOMA, waist circumference, body mass index and blood pressure were found, but the HDL-cholesterol correlation coefficients were negative (data not shown). The highest correlation was between cholesterol and LDL-cholesterol (0.83), waist and body mass index (0.80), and systolic and diastolic blood pressure (0.66). A high correlation was also seen between fasting glucose and HOMA $(0.38)$ and glucose and triglyceride (0.26), body mass index and blood pressure (0.36).

As shown in Table 2, the exploratory factor analysis revealed three principle factors that combined, explained $68 \%$ of the overall variance. Factor 1 explained $30 \%$ of the variance and had large factor loading for blood pressure, waist and body mass index, and thus can be interpreted as a "blood pressure/ obesity" factor. Factor 2 explained $22 \%$ of the variance and had large factor loadings for total cholesterol and LDL-cholesterol, and thus was interpreted as "cholesterol" factor. Factor 3 was characterized by large factor loadings in HOMA, glucose, triglyceride and HDLcholesterol, which explained $17 \%$ of the variance, and thus can be interpreted as "insulin resistance/dyslipidemia" factor.

The intra-trait familial correlation of various quantitative factors between spouses, parent-offspring and siblings, and the heritability estimation by variance components analysis are presented in Table 3. Sibling correlations were higher than parent-offspring correlations, and both were much higher than spouses, indicating high genetic components of these quantitative components. The highest correlation was seen in blood pressure/obesity factor in sibling (0.34), then

Characteristics of various metabolic syndrome and insulin resistance components among family subjects, specified by generation and gender

\begin{tabular}{|c|c|c|c|c|c|c|c|c|c|c|c|c|}
\hline & \multicolumn{2}{|c|}{$\begin{array}{l}\text { Grandfather } \\
(N=82)\end{array}$} & \multicolumn{2}{|c|}{$\begin{array}{l}\text { Grandmother } \\
(N=123)\end{array}$} & \multicolumn{2}{|c|}{$\begin{array}{l}\text { Father } \\
(N=218)\end{array}$} & \multicolumn{2}{|c|}{$\begin{array}{l}\text { Mother } \\
(N=230)\end{array}$} & \multicolumn{2}{|c|}{$\begin{array}{l}\text { Sons } \\
(N=342)\end{array}$} & \multicolumn{2}{|c|}{$\begin{array}{l}\text { Daughter } \\
(N=361)\end{array}$} \\
\hline & Mean & S.D. & Mean & S.D. & Mean & S.D. & Mean & S.D. & Mean & S.D. & Mean & S.D. \\
\hline Age (years) & 71.8 & 7.3 & 70.0 & 7.9 & 46.1 & 6.1 & 41.3 & 4.3 & 16.9 & 2.6 & 17.3 & 2.5 \\
\hline Cholesterol (mg/dL) & 195.4 & 37.0 & 216.7 & 40.3 & 201.2 & 34.3 & 190.7 & 36.5 & 160.5 & 32.1 & 173.2 & 36.5 \\
\hline Triglyceride (mg/dL) & 123.5 & 73.1 & 156.1 & 81.8 & 197.0 & 156.1 & 116.0 & 91.5 & 85.8 & 42.5 & 88.1 & 41.1 \\
\hline HDL-cholesterol (mg/dL) & 40.2 & 12.0 & 41.8 & 10.2 & 40.4 & 10.9 & 44.1 & 10.7 & 43.5 & 10.7 & 44.1 & 9.4 \\
\hline LDL-cholesterol (mg/dL) & 125.9 & 33.1 & 140.9 & 39.1 & 126.5 & 36.4 & 118.2 & 36.0 & 75.9 & 31.4 & 88.8 & 34.9 \\
\hline Fasting glucose (mg/dL) & 112.1 & 27.0 & 129.8 & 48.2 & 121.3 & 37.6 & 111.8 & 32.1 & 118.81 & 18.8 & 106.5 & 14.8 \\
\hline HOMA & 7.7 & 37.6 & 3.4 & 3.3 & 8.6 & 43.2 & 2.1 & 4.1 & 2.3 & 2.8 & 2.3 & 1.3 \\
\hline Waist circumference $(\mathrm{cm})$ & 84.6 & 12.5 & 85.9 & 9.5 & 84.3 & 10.4 & 75.1 & 10.2 & 67.1 & 10.9 & 64.6 & 9.1 \\
\hline Body mass index $\left(\mathrm{kg} / \mathrm{m}^{2}\right)$ & 23.5 & 3.3 & 25.7 & 3.9 & 24.4 & 3.8 & 24.1 & 4.0 & 20.2 & 3.6 & 20.5 & 3.5 \\
\hline Systolic BP (mmHg) & 130.5 & 20.6 & 137.5 & 20.1 & 121.2 & 13.7 & 116.1 & 14.7 & 112.2 & 12.9 & 107.1 & 11.5 \\
\hline Diastolic BP (mmHg) & 79.5 & 13.2 & 81.7 & 11.8 & 81.2 & 10.9 & 75.9 & 10.5 & 69.5 & 9.6 & 68.0 & 8.7 \\
\hline Metabolic syndrome numbers & 2.14 & 1.26 & 3.25 & 1.37 & 2.32 & 1.42 & 1.77 & 1.27 & 1.50 & 1.20 & 1.20 & 1.11 \\
\hline
\end{tabular}


Table 2

Results of adjusted ${ }^{\mathrm{a}}$ factor component and variance components among various metabolic syndrome and insulin resistance components in the study population $(N=1227)$

\begin{tabular}{lccc}
\hline & $\begin{array}{l}\text { Factor 1: blood } \\
\text { pressure/obesity }\end{array}$ & $\begin{array}{l}\text { Factor 2: } \\
\text { cholesterol }\end{array}$ & $\begin{array}{r}\text { Factor 3: insulin } \\
\text { resistance/dyslipidemia }\end{array}$ \\
\hline Systolic blood pressure & 0.83 & 0.12 & 0.06 \\
Diastolic blood pressure & 0.84 & 0.12 & 0.01 \\
Waist circumference & 0.77 & 0.32 & 0.19 \\
Body mass index & 0.74 & 0.27 & 0.24 \\
Cholesterol & 0.14 & 0.96 & 0.03 \\
LDL-cholesterol & 0.32 & 0.87 & 0.08 \\
HOMA & 0.04 & 0.01 & 0.78 \\
Fasting glucose & 0.04 & 0.21 & 0.71 \\
Triglyceride & 0.41 & 0.44 & 0.45 \\
HDL-cholesterol & -0.38 & 0.19 & -0.48 \\
Percent total variance $(\%)$ & 29.8 & 21.6 & \\
Percent cumulative variance $(\%)$ & 29.8 & 51.4 & 68.0 \\
\hline
\end{tabular}

\footnotetext{
${ }^{\mathrm{a}}$ Adjusted for sex and age; three factors were retained by the criterion of eigenvalue more than 1 .
}

BMI in parent-offspring and waist in siblings (0.30). Most sibling and parent-offspring correlation coefficients were more than 0.2 , while only spouse waist correlation was more than 0.2 , indicating assortive matching in waist circumference. All heritability values among quantitative traits were significant. The estimated heritability was the highest in LDL and cholesterol factor $(0.36$ and 0.40$)$, then blood pressure/obesity factor (0.27), and insulin resistance/ dyslipidemia (0.27). There were significant heritability estimates among HDL-cholesterol (0.30), fasting glucose (0.27), systolic blood pressure (0.32) and diastolic blood pressure $(0.23)$. The lowest heritability was for waist (0.17).
Contingency tables of affected status of MetS, specified by numbers of siblings are presented in Table 4. We found significant familial aggregation of MetS status among siblings and relatives; higher MetS prevalence rates were found among proband's MetS status. The patterns among relatives were similar as siblings. Recurrence risk ratios among siblings and relatives were 2.95 (95\% confidence interval [CI]: 1.39_ 6.26) and 2.48 (95\% CI: $1.77-3.48)$, respectively.

Table 5 shows that GEE model for predicting odds ratio for MetS status among family relatives. After adjusting age, gender, smoking, and alcohol drinking status, the odds ratio of proband's MetS status decreased to 1.99 (95\% CI: $1.08-3.66, P=0.026)$. The adjusted

Table 3

Family intra-trait correlations $(r)$, equal weight to pedigree, and estimated heritability of various metabolic syndrome and insulin resistance components in the study population

\begin{tabular}{|c|c|c|c|c|c|}
\hline & Spouse $\left(160^{\mathrm{a}}\right)$ & Parental offspring $\left(904^{\mathrm{a}}\right)$ & Sibling $\left(452^{\mathrm{a}}\right)$ & Heritability & S.E. \\
\hline Cholesterol & 0.12 & 0.23 & 0.20 & 0.328 & 0.061 \\
\hline Triglyceride & 0.01 & 0.20 & 0.22 & 0.267 & 0.067 \\
\hline HDL-cholesterol & 0.06 & 0.25 & 0.24 & 0.299 & 0.061 \\
\hline LDL-cholesterol & 0.05 & 0.28 & 0.23 & 0.401 & 0.058 \\
\hline Fasting glucose & 0.17 & 0.07 & 0.10 & 0.272 & 0.067 \\
\hline HOMA & 0.10 & 0.16 & 0.10 & 0.255 & 0.060 \\
\hline Waist & 0.21 & 0.28 & 0.30 & 0.174 & 0.075 \\
\hline Body mass index & 0.01 & 0.30 & 0.29 & 0.216 & 0.073 \\
\hline Systolic blood pressure & 0.03 & 0.16 & 0.26 & 0.321 & 0.057 \\
\hline Diastolic blood pressure & 0.07 & 0.16 & 0.24 & 0.232 & 0.078 \\
\hline Factor 1: blood pressure/obesity & 0.02 & 0.11 & 0.34 & 0.275 & 0.051 \\
\hline Factor 2: cholesterol & 0.15 & 0.26 & 0.22 & 0.361 & 0.050 \\
\hline Factor 3: insulin resistance/dyslipidemia & 0.15 & 0.18 & 0.18 & 0.266 & 0.056 \\
\hline
\end{tabular}

S.E.: standard error of heritability.

${ }^{a}$ Pair numbers. 
Table 4

Sibling recurrence risk ratios of metabolic syndrome status, specified by proband's status of metabolic syndrome, in the study population (estimated odds ratio: 2.95 , 95\% confidence interval: 1.39-6.26, $P=0.005$, by logistic regression model)

\begin{tabular}{|c|c|c|c|c|c|c|c|}
\hline \multirow[t]{2}{*}{ Family size } & \multicolumn{6}{|c|}{ Affected sibling numbers } & \multirow[t]{2}{*}{ Total siblings } \\
\hline & 0 & & & 2 & & 3 & \\
\hline \multicolumn{8}{|c|}{ Proband metabolic syndrome status $(+) N=56$} \\
\hline 1 & 19 & & 4 & & & & 23 \\
\hline 2 & 4 & & 8 & 0 & & & 12 \\
\hline 3 & 2 & & 1 & 0 & & ) & 3 \\
\hline Total siblings & 33 & & 3 & 0 & & 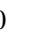 & 56 \\
\hline \multirow[t]{2}{*}{ Family size } & \multicolumn{6}{|c|}{ Affected sibling numbers } & Total siblings \\
\hline & 0 & 1 & 2 & 3 & 4 & 5 & \\
\hline \multicolumn{8}{|c|}{ Proband metabolic syndrome status (-) $N=247$} \\
\hline 1 & 75 & 8 & & & & & 83 \\
\hline 2 & 45 & 9 & 0 & & & & 54 \\
\hline 3 & 9 & 4 & 0 & 0 & & & 13 \\
\hline 4 & 2 & 1 & 0 & 0 & 0 & & 3 \\
\hline 5 & 0 & 1 & 0 & 0 & 0 & 0 & 1 \\
\hline Total siblings & 200 & 47 & 0 & 0 & 0 & 0 & 247 \\
\hline
\end{tabular}

odds ratios of 3 principal factors for predicting MetS were all significant, with highest odds ratio in blood pressure/obesity factor (OR: 1.27, 95\% CI: 1.22-1.33), then insulin resistance/dyslipidemia (OR: $1.19,95 \%$ CI: 1.16-1.23). And the odds ratio of cholesterol was the least predictor for familial MetS (OR: $1.11,95 \%$ CI: $1.08-1.14)$.

Table 5

Adjusted odds ratio and 95\% confidence intervals for predicting the metabolic syndrome status among family relatives

\begin{tabular}{lllll}
\hline Variables & $\begin{array}{l}\text { Odds } \\
\text { ratio }\end{array}$ & $\begin{array}{l}\text { Lower } \\
95 \% \text { CI }\end{array}$ & $\begin{array}{l}\text { Upper } \\
95 \% \text { CI }\end{array}$ & $P$ value \\
\hline Age (+1 year) & 1.00 & 0.98 & 1.01 & 0.545 \\
$\begin{array}{l}\text { Proband metabolic } \\
\quad \text { syndrome: yes vs. no }\end{array}$ & 1.99 & 1.08 & 3.66 & 0.026 \\
$\begin{array}{l}\text { Female vs. male } \\
\text { Smoke status: }\end{array}$ & 1.05 & 0.59 & 1.89 & 0.857 \\
$\quad$ yes vs. no & 0.44 & 0.23 & 0.85 & 0.014 \\
$\begin{array}{l}\text { Drinking status: } \\
\quad \text { yes vs. no }\end{array}$ & 1.42 & 0.80 & 2.52 & 0.234 \\
$\begin{array}{l}\text { Factor 1: blood } \\
\text { pressure/obesity }\end{array}$ & 1.27 & 1.22 & 1.33 & $<0.0001$ \\
$\begin{array}{l}\text { Factor 2: cholesterol } \\
\begin{array}{l}\text { Factor 3: insulin } \\
\text { resistance/dyslipidemia }\end{array}\end{array}$ & 1.11 & 1.08 & 1.14 & $<0.0001$ \\
& & 1.16 & 1.23 & $<0.0001$ \\
\hline
\end{tabular}

GEE model was used to adjust familial correlation and size effects. Three independent principal component factors to predict metabolic syndrome status among family. Three factors were standardized as mean 100 and standard deviation as 10 .

\section{Discussion}

This study, to authors' knowledge, is the first report to use a genetic epidemiology approach to dissect the genetic components, heritability and recurrence risk ratio of MetS and lipid-related quantitative components on ethnic Chinese community, based on the adolescent probands. We demonstrated significant familial correlation and heritability lipid-related quantitative components, and the recurrence risk ratios of MetS were more than two-fold among family members. The study results strongly suggest the presence of genetic susceptibility on MetS; also, the blood pressure/obesity factor predicts most significantly MetS among population.

Previous studies on MetS also showed significant familial correlation and heritability among different subjects $[7,8]$. This study had several advantages. First, the study subjects ascertained from homogeneous and young adolescent proband families can expel adulthood environmental confounding factors and other systemic diseases [2]. Diseased elderly patients from hospital composed more lifestyle factors affecting MetS and might decrease the power to detect genetic components. Second, three independent factors were clearly defined from MetS and lipid-related variables, and we can estimate the independent effect of each factor for MetS. Also, we incorporated statistical methods such as GEE to tackle the dependence among family members. Due to multiple risk factors clustering, MetS is a difficult phenotype to dissect underlying genetic factors. Finally, we specified binary and continuous traits of MetS, and estimated recurrence risk ratios among siblings and relatives. Sibling recurrence risk ratio can provide good hints for genetic components [9]. In this study, we clearly demonstrated significant sibling recurrence risk ratio and the results implied that genetic components existed among Chinese population.

Although previous reports on large-scale genomewide linkage analyses to locating genes for MetS were reported in the locus of chromosomes $1 \mathrm{q}$ and $3 \mathrm{q}$ [20-25] and numerous candidate gene markers were proven to be associated with MetS and insulin resistance components [26-30], heritability and recurrence risk ratio estimation still be unknown, especially for young generation. In this study, we clearly quantified the effects of genetic components on MetS and its components. In particular, we specified three independent factors to prevent multi-collinearity problem for statistical inference. Factor analysis provided an important tool for classifying underlying factors for metabolic syndrome, and we found blood pressure/ obesity factor seemed to be most important factor for 
MetS among family members. There were interrelationships among lipid, blood pressure, obesity and insulin resistance indices. Because of possible pleiotropic effects of metabolic syndrome trait components, it is difficult to dissect these components into separate independent factors.

Central obesity was viewed as a central factor for MetS. Urbanization and globalization accelerated the obesity endemic among the non-Western countries [31]. Our study also demonstrated a high prevalence of central obesity among adolescents and their families among ethnic Chinese. Weight control should be considered as the first priority for MetS prevention program in the area of rapid economic progress, such as in Asia-Pacific countries. Also, among Pacific Asian countries, hypertension was associated more with stroke, and dyslipidemia more with coronary heart disease. In urban Chinese, cholesterol levels increased progressively [32]. Our study proved that these cholesterol and insulin resistance factors had significant genetic components and familial aggregation. Familybased lifestyle intervention on body weight control and physical activity might be useful for health promotion among community population.

The study limitations were following. First, we did not include all family members such as grandparents in the study. There were only $14 \%$ grandparents in the study. Large pedigree structure provides more genetic transmission information than nuclear family, and potential selection bias would invalidate the results. Also, the response rates of the parents were $59 \%$, and up to a quarter of the parents' data were missed in the study. It would reduce the power for estimating spouse correlations. In all participants, there were average 4.2 persons included in one family. Also, the counts for each family member were plotted in the following figure. We collected the siblings up to 703 subjects, and we consider the results of the parent-offspring and sibling correlations are valid. Second, we did not collect dietary habits and physical activity intensity in the lifestyle habits, and only smoking and alcohol drinking were recorded. The physical activity and dietary factors were important determinants for metabolic syndrome. Third, we cannot differentiate common environmental from pure genetic factors in this study. Familial aggregation of metabolic syndrome had complex interaction among genes and environmental factors.

In conclusion, we clearly demonstrated familial aggregation and recurrence risk ratio of metabolic syndrome and lipid components among the general ethnic Chinese population in Taiwan.

\section{Acknowledgements}

This work was partly supported by grants from the National Council of Science (NSC 93-2314-B-002-218, NSC 92-2314-B-002-197), and the funds of National Taiwan University Hospital (NTUH 93S023), Taipei, Taiwan. We sincerely thank all of the Chin-Shan community participants for their cooperation and the support of the Cardiologists in the Department of Internal Medicine, National Taiwan University Hospital, Taiwan.

\section{References}

[1] K.G. Alberti, P.Z. Zimmet, Definition, diagnosis and classification of diabetes mellitus and its complications. Part 1. Diagnosis and classification of diabetes mellitus provisional report of a WHO consultation, Diabetic. Med. 15 (1998) 539-553.

[2] W.J. Chen, P.H. Liu, Y.Y. Ho, K.L. Chien, M.T. Lo, W.L. Shih, et al., Sibling recurrence risk ratio analysis of the metabolic syndrome and its components over time, BMC Genet. 4 (Suppl. 1) (2003) $\mathrm{S} 33-\mathrm{S} 38$.

[3] E.S. Ford, W.H. Giles, W.H. Dietz, Prevalence of the metabolic syndrome among US adults: findings from the third National Health and Nutrition Examination Survey, JAMA 287 (2002) 356-359.

[4] J.A. Al Lawati, A.J. Mohammed, H.Q. Al Hinai, P. Jousilaht, Prevalence of the metabolic syndrome among Omani adults, Diabetes Care 26 (2003) 1781-1785.

[5] A. Ramachandran, C. Snehalatha, K. Satyavani, S. Sivasankari, V. Vijay, Metabolic syndrome in urban Asian Indian adults-a population study using modified ATP III criteria, Diabetes Res. Clin. Pract. 60 (2003) 199-204.

[6] D.A. Tregouet, B. Herbeth, I. Juhan-Vague, G. Siest, P. Ducimetiere, L. Tiret, Bivariate familial correlation analysis of quantitative traits by use of estimating equations: application to a familial analysis of the insulin resistance syndrome, Genet. Epidemiol. 16 (1999) 69-83.

[7] P. Poulsen, A. Vaag, K. Kyvik, H. Beck-Nielsen, Genetic versus environmental aetiology of the metabolic syndrome among male and female twins, Diabetologia 44 (2001) 537-543.

[8] M.A. Austin, K.L. Edwards, M.J. McNeely, W.L. Chandler, D.L. Leonetti, P.J. Talmud, Heritability of multivariate factors of the metabolic syndrome in nondiabetic Japanese Americans, Diabetes 53 (2004) 1166-1169.

[9] N. Risch, K. Merikangas, The future of genetic studies of complex human diseases, Science 273 (1996) 1516-1517.

[10] K.L. Chien, H.C. Hsu, T.C. Su, Y.T. Lee, Consistency in genetic inheritance mode and heritability patterns of triglyceride vs. high density lipoprotein cholesterol ratio in two Taiwanese family samples, BMC J.: Genet. 4 (2003) 7-16.

[11] K.L. Chien, W.J. Chen, H.C. Hsu, T.C. Su, M.F. Chen, Y.T. Lee, Major gene effects in systolic and diastolic blood pressure in the families receiving health examination in Taiwan, J. Hypertens. 21 (2003) 1-7.

[12] Y.T. Lee, R.S. Lin, F.C. Sung, C.Y. Yang, K.L. Chien, W.J. Chen, et al., Chin-Shan community cardiovascular cohort in Taiwan: baseline data and 5-year follow-up morbidity and mortality, J. Clin. Epidemiol. 53 (2000) 836-846. 
[13] L. Andersen, B. Dinesen, P.N. Jorgensen, F. Poulser, M.E. Roder Enzyme immunoassay for intact human insulin in serum or plasma, Clin. Chem. 39 (1993) 578-582.

[14] Executive summary of the third report of the National Cholesterol Education Program (NCEP) expert panel on detection, evaluation, and treatment of high blood cholesterol in adults (adult treatment panel III), JAMA 285 (2001) 2486-2497.

[15] C.E. Tan, S. Ma, D. Wai, S.K. Chew, E.S. Tai, Can we apply the National Cholesterol Education Program Adult Treatment Panel definition of the metabolic syndrome to Asians? Diabetes Care 27 (2004) 1182-1186.

[16] S. Cook, M. Weitzman, P. Auinger, M. Nguyen, W.H. Dietz, Prevalence of a metabolic syndrome phenotype in adolescents: findings from the third National Health and Nutrition Examination Survey, 1988-1994, Arch. Pediatr. Adolesc. Med. 157 (2003) 821-827.

[17] S.A.G.E., Statistical analysis for genetic epidemiology, Release 3.1. Computer program package available from the Department of Epidemiology and Biostatistics, Rammelkamp Center for Education and Research, MetroHealth Campus, Case Western Reserve University, Cleveland, 1997.

[18] L. Almasy, J. Blangero, Multipoint quantitative-trait linkage analysis in general pedigrees, Am. J. Hum. Genet. 62 (1998) 1198-1211.

[19] K.Y. Liang, S.L. Zeger, Regression analysis for correlated data, Annu. Rev. Public. Health 14 (1993) 43-68.

[20] S. Francke, M. Manraj, C. Lacquemant, C. Lecoeur, F. Lepretre, P. Passa, et al., A genome-wide scan for coronary heart disease suggests in Indo-Mauritians a susceptibility locus on chromosome $16 \mathrm{p} 13$ and replicates linkage with the metabolic syndrome on 3q27, Hum. Mol. Genet. 10 (2001) 2751-2765.

[21] C.D. Langefeld, L.E. Wagenknecht, J.I. Rotter, A.H. Williams, J.E. Hokanson, M.F. Saad, et al., Linkage of the metabolic syndrome to 1q23-q31 in Hispanic families: the insulin resistance atherosclerosis study family study, Diabetes 53 (2004) $1170-1174$.

[22] R.J. Loos, P.T. Katzmarzyk, D.C. Rao, T. Rice, A.S. Leon, J.S. Skinner, et al., Genome-wide linkage scan for the metabolic syndrome in the HERITAGE family study, J. Clin. Endocrinol. Metab. 88 (2003) 5935-5943.
[23] A.H. Kissebah, G.E. Sonnenberg, J. Myklebust, M. Goldstein, K. Broman, R.G. James, et al., Quantitative trait loci on chromosomes 3 and 17 influence phenotypes of the metabolic syndrome, Proc. Natl. Acad. Sci. U.S.A. 1997 (2000) 14478-14483.

[24] M.C. Ng, W.Y. So, V.K. Lam, C.S. Cockram, G.I. Bell, N.J. Cox, et al., Genome-wide scan for metabolic syndrome and related quantitative traits in Hong Kong Chinese and confirmation of a susceptibility locus on chromosomes1q21-25, Diabetes 53 (2004) 2676-2683.

[25] K. Xiang, Y. Wang, T. Zheng, W. Jia, J. Li, L. Chen, et al., Genome-wide search for type 2 diabetes/impaired glucose homeostasis susceptibility genes in the Chinese: significant linkage to chromosome 6q21-q23 and chromosome 1q21q24, Diabetes 53 (2004) 228-234.

[26] C. Lacquemant, P. Froguel, S. Lobbens, P. Izzo, C. Dina, J. Ruiz, The adiponectin gene SNP +45 is associated with coronary artery disease in Type 2 (non-insulin-dependent) diabetes mellitus, Diabetes Med. 21 (2004) 776-781.

[27] S. Martin, V. Nicaud, S.E. Humphries, P.J. Talmud, Contribution of APOA5 gene variants to plasma triglyceride determination and to the response to both fat and glucose tolerance challenges, Biochim. Biophys. Acta 1637 (2003) 217-225.

[28] W.S. Yang, C.A. Hsiung, L.T. Ho, Y.T. Chen, C.T. He, J.D. Curb, et al., Genetic epistasis of adiponectin and PPARgamma2 genotypes in modulation of insulin sensitivity: a family-based association study, Diabetologia 46 (2003) 977-983.

[29] J. Sanchez-Corona, S.E. Flores-Martinez, M.V. Machorro-Lazo, C. Galaviz-Hernandez, M.C. Moran-Moguel, F.J. Perea, et al., Polymorphisms in candidate genes for type 2 diabetes mellitus in a Mexican population with metabolic syndrome findings, Diabetes Res. Clin. Pract. 63 (2004) 47-55.

[30] H. Wang, H. Zhang, Y. Jia, Z. Zhang, R. Craig, X. Wang, et al., Adiponectin receptor 1 gene (ADIPOR 1 ) as a candidate for type 2 diabetes and insulin resistance, Diabetes 53 (2004) 2132-2136.

[31] K.S. Reddy, Cardiovascular disease in non-Western countries, N. Engl. J. Med. 350 (2004) 2438-2440.

[32] J. He, D. Gu, K. Reynolds, X. Wu, P. Muntner, J. Zhao, et al., Serum total and lipoprotein cholesterol levels and awareness, treatment, and control of hypercholesterolemia in China, Circulation 110 (2004) 405-411. 